\title{
Cortical and Subcortical Connectivity Changes during Decreasing Levels of Consciousness in Humans: A Functional Magnetic Resonance Imaging Study using Propofol
}

\author{
Róisín Ní Mhuircheartaigh, Debbie Rosenorn-Lanng, Richard Wise, Saad Jbabdi, Richard Rogers, and Irene Tracey \\ Centre for Functional Magnetic Resonance of the Brain (FMRIB), Department of Clinical Neurology and Nuffield Department of Anaesthetics, John \\ Radcliffe Hospital, University of Oxford, Oxford, OX3 9DU, United Kingdom
}

While ubiquitous, pharmacological manipulation of consciousness remains poorly defined and incompletely understood (Prys-Roberts, 1987). This retards anesthetic drug development, confounds interpretation of animal studies conducted under anesthesia, and limits the sensitivity of clinical monitors of cerebral function to intact perception. Animal and human studies propose a functional "switch" at the level of the thalamus, with inhibition of thalamo-cortical transmission characterizing loss of consciousness (Alkire et al., 2000; Mashour, 2006). We investigated the effects of propofol, widely used for anesthesia and sedation, on spontaneous and evoked cerebral activity using functional magnetic resonance imaging (fMRI). A series of auditory and noxious stimuli was presented to eight healthy volunteers at three behavioral states: awake, "sedated" and "unresponsive." Performance in a verbal task and the absence of a response to verbal stimulation, rather than propofol concentrations, were used to define these states clinically. Analysis of stimulus-related blood oxygenation level-dependent signal changes identified reductions in cortical and subcortical responses to auditory and noxious stimuli in sedated and unresponsive states. A specific reduction in activity within the putamen was noted and further investigated with functional connectivity analysis. Progressive failure to perceive or respond to auditory or noxious stimuli was associated with a reduction in the functional connectivity between the putamen and other brain regions, while thalamo-cortical connectivity was relatively preserved. This result has not been previously described and suggests that disruption of subcortical thalamo-regulatory systems may occur before, or even precipitate, failure of thalamo-cortical transmission with the induction of unconsciousness.

\section{Introduction}

Controlled reversible pharmacological manipulations of consciousness are routine in medical practice, e.g., general anesthesia. Neither a unified theory to explain these phenomena nor universally accepted behavioral or neurophysiological definitions exist (Prys-Roberts, 1987). Relative dose-response relationships of the associated immobility, amnesia and analgesia are drug specific and difficult to disentangle neuroscientifically, as they may result from drug activity at different sites (Antognini and Schwartz, 1993).

Specific drug-receptor interactions (Sear, 2009) at sites including GABA $\left(\mathrm{GABA}_{\mathrm{A}}\right)$ receptors (Nelson et al., 2002), 2-pore-domain potassium channels, and $\mathrm{N}$-methyl-D-aspartate (NMDA) receptors (Franks, 2008) are now recognized and have replaced previous theories of generalized phospholipid bilayer disruption (Meyer, 1899). It is perhaps intuitive, linking these molecular mechanisms to network effects and ultimately behavior, to envisage an anatomical site

Received Nov. 8, 2009; revised April 15, 2010; accepted May 10, 2010

We thank the Medical Research Council of Great Britain and Northern Ireland (FMRIB Centre), the International Anesthesia Research Society and Association of Anesthetists of Great Britain and Ireland (R.Ním., financial research support), and Dr. Katy Vincent and Dr. Catherine Warnaby for their invaluable assistance throughout.

Correspondence should be addressed to Dr. Róisín Ní Mhuircheartaigh, Centre for Functional Magnetic Resonance of the Brain (FMRIB), Department of Clinical Neurology and Nuffield Department of Anaesthetics, John Radcliffe Hospital, University of Oxford, 0xford, 0X3 9DU, UK. E-mail: roisin@fmrib.ox.ac.uk.

DOI:10.1523/JNEUROSCI.5516-09.2010

Copyright $\odot 2010$ the authors $\quad 0270-6474 / 10 / 309095-08 \$ 15.00 / 0$ acting as a gateway or "switch," permitting or preventing cortical processing. Identification of such a switch has not been straightforward. Molecular, animal, and human studies have converged upon disruption of thalamocortical communication as a key component of anesthetic-induced unconsciousness (Angel, 1991; Antognini and Schwartz, 1993; Devor and Zalkind, 2001; Mashour, 2006). The reticular and intralaminar thalamic nuclei have been specifically implicated in both pathologies of consciousness and pharmacodynamic phenomena (Alkire et al., 1999, 2007; Detsch et al., 1999; Van der Werf et al., 2002; Schiff, 2008). Several authors have suggested, however, that changes within the thalamus could be a consequence of deafferentation rather than necessarily causal to anesthesia (Schiff and Plum, 2000; Alkire, 2008). Alternatively, the unconscious state may arise from uncoupling of corticocortical connections or "cognitive unbinding" (Massimini et al., 2005; Mashour, 2006). Recently, the picture has been further complicated by evidence from primates showing preservation of resting state networks, coherent system level neural oscillations, during isoflurane anesthesia (Biswal et al., 1995; Gusnard and Raichle, 2001; Vincent et al., 2007). In short, there is substantial evidence that rather than producing global suppression, anesthesia affects the function of discrete networks (White and Alkire, 2003; Laitio et al., 2007).

Positron emission tomography (PET) has informed much of our understanding of the network-level activity of both volatile and intravenous anesthetics in human subjects (Alkire et al., 1999; Kaisti et al., 2002; Veselis et al., 2004; Laitio et al., 2009). 
Specific relative reductions in thalamic and cortical blood flow occur in sedated and anesthetized states. Fiset et al. (1999) found that propofol preferentially decreased regional cerebral blood flow (rCBF) in the thalamus, midbrain, cuneus, precuneus, posterior cingulate, and orbitofrontal cortex. Bonhomme et al. (2001) found that propofol reduced rCBF changes during vibrotactile stimulation at doses below those required to suppress the thalamic rCBF response. We aimed to extend these important findings, harnessing the superior temporal resolution of functional magnetic resonance imaging (fMRI) to test the hypothesis that decreases in functional connectivity occur with deepening sedation. Based on existing PET and animal literature, we focused on connectivity among the thalamus, cortical areas, and thalamoregulatory regions, including the basal ganglia.

\section{Materials and Methods}

The study was approved by the Local Research Ethics Committee (Central Oxfordshire Research Ethics Committee, Oxford, Oxfordshire, UK) and complied with the guidelines of the Declaration of Helsinki (1996). Fully informed written consent was obtained.

Study design. The volunteers in this study participated in two experimental sessions, at least 1 week apart, and informed consent was independently obtained before each session. Each subject completed the experimental protocol twice, first in a physiology laboratory, then again in the fMRI scanner. The purpose of the prescanning laboratory session was twofold: to ensure that subjects would safely tolerate the administration of propofol and to estimate the propofol effect site concentrations required to produce the designated clinical sedation endpoints during the subsequent fMRI session.

Volunteers. Eight right-handed healthy volunteers, four males and four females, between the ages of 21 and 37 (mean 28.8) were recruited to participate in the study. All subjects were medically screened. They were physically healthy and had no history of psychiatric illness. They were excluded if they were taking medication or drugs acting on the CNS for therapeutic or recreational use. A medical history and, where appropriate, physical examination was performed to ensure they were fit and well (American Society Of Anesthesiology grade I) and that there were no contraindications to the administration of propofol or to magnetic resonance imaging.

Physiological monitoring. Both the physiology laboratory and the fMRI scanner were equipped for the administration of sedative medication in compliance with the minimum monitoring guidelines of the Association of Anesthetists of Great Britain and Ireland (Recommendations for standards of monitoring during anesthesia and recovery, Ed 4, The Association of Anesthetists of Great Britain and Ireland, London, UK, March 2007).

The subjects were monitored continuously during both the prescanning and scanning sessions. Heart rate, noninvasive blood pressure, end tidal carbon dioxide concentration and oxygen saturation were measured continuously using a 9500 Multigas Monitor (MR Equipment Corporation; UK supplier Wardray Premise Ltd). During both sessions subject physiology was recorded both manually and electronically.

Drug administration. Volunteers adhered to a standard preanesthesia protocol: they were asked not to take fluids for $2 \mathrm{~h}$ and solids for $6 \mathrm{~h}$ before both experimental sessions. They were supervised until fully recovered, $\sim 2 \mathrm{~h}$ after the propofol infusion was stopped. Anesthesia day case discharge criteria were met and appropriate information was provided.

Intravenous access was established (23G Wallace Y-Can, SIMS Portex Ltd) and standard monitoring instituted (pulse oximetry, electrocardiography and noninvasive blood pressure measurement). Throughout the experiments oxygen was administered at $2 \mathrm{~L} \mathrm{~min}^{-1}$ via nasal cannulae (Adult Nasal Cannula with modified nasal prongs, Salter Labs Ref 4707), which allow simultaneous oxygen delivery and sampling of expired airway gases for capnography. A dedicated senior anesthesiologist was responsible for the administration of propofol and monitoring of physiological parameters throughout both sessions. The anesthesiologist was not otherwise involved in the conduct of the experiment and would stop the experiment if they had any concerns over the safety of the volunteer.

A $1 \%$ propofol solution (Diprivan, AstraZeneca UK Limited) was administered using a target-controlled infusion (TCI) pump. A Graseby 3500 pump (SIMS Graseby Ltd) programmed with a Diprifusor module (AstraZeneca UK Limited). This uses the Marsh modification (Marsh et al., 1991) of the pharmacokinetic model described by Gepts et al. (1987). Effect site concentrations of propofol predicted from the Diprifusor model are reported here.

The target concentration was set at an initial value of $1.0 \mu \mathrm{g} / \mathrm{ml}$ and tests of cognitive impairment were made (see below). Using these results, the effect site concentration was adjusted until the defined clinical endpoints were achieved. A 10 min pause allowed equilibration after each state change was established, before the experiment proceeded.

Determination of levels of sedation. For each subject, at each session, the level of propofol required to produce two levels of impaired consciousness was identified. A lower level (sedated), was defined for our experiment as significant impairment in the performance of a verbal task; the higher level (unresponsive) was defined as loss of verbal contact.

During a number of pilot sessions we assessed and modified a number of tests to develop a simple task suitable for use in the fMRI scanner that reproducibly measured the cognitive impairment caused by propofol. Our test involved timing the volunteer while reciting the letters of the alphabet, the days of the week and then the months of the year in sequence. The individual's sedated state was defined as the propofol dose at which the verbal task was prolonged by $30 \%$ above baseline (awake), with a 10\% tolerance. The "unresponsive" propofol level was taken as the level at which the volunteer no longer responded to verbal prompting.

Loss of verbal contact was deliberately chosen as an end point as this is easily defined, testable, and reproducible. Loss of verbal contact is used in the United Kingdom to define the transition from sedation to anesthesia (General Dental Council, Maintaining Standards, November, 1997).

At the prescanning session the predicted plasma concentration of propofol was determined individually for each subject for the different cognitive states. The doses determined during the first session were used as a guide for the scanning session. The predicted plasma concentration was then adjusted to again achieve the defined states. As the study levels were defined by clinical state rather than drug level, if a subject was no longer responsive to verbal command at the guide level determined at the bench session for sedation, the order of the experiment was reversed so that the data were collected for the higher propofol dose and then the infusion was reduced until the criteria for the "sedated" state were met. This occurred in five of the eight subjects.

Paradigm design. Noxious stimuli were delivered by a purpose built, in-house designed thermode, applied to the dorsum of the left hand. Thresholding was performed using heat stimuli, administered for $3 \mathrm{~s}$ each, at increasing temperatures until the temperature that reliably produced a rating of $7 / 10$ on an 11 point numerical rating scale was established. Twelve of these noxious thermal stimuli (i.e., at the temperature rated by the subject as $7 / 10$ ) were delivered during the experiment. Subjects were asked to verbally rate the average intensity of these 12 stimuli on the same scale at the end of the "awake" and "sedated" blocks.

A list of 12 words was repeated three times at each propofol dose. Different wordlists were presented at each state of consciousness. Playing this wordlist backwards generated meaningless sounds, acoustically similar (regarding volume, frequency distribution and duration) to the wordlist. The full auditory paradigm consisted therefore of words, reversed words and an additional 12 computer generated pure tones. The 12 thermal pain stimuli were interleaved with the auditory stimuli. Auditory stimuli were presented binaurally with magnetic resonance-compatible electrostatic headphones (MRC Institute of Hearing Research). Volume adequacy was checked before the experiment began.

The word lists were also used as the basis of a memory test (Rasmusson et al., 1995; Shapiro et al., 1999). At the end of each paradigm presentation, the subjects were presented with a list of 24 words, the original 12 words and 12 new words, in a random order and asked to identify which of the words they had previously heard. The false-positive count was subtracted from the true-positive count to yield a "discrimination index" ranging from +12 to -12 with an expected score of 0 on guessing. 

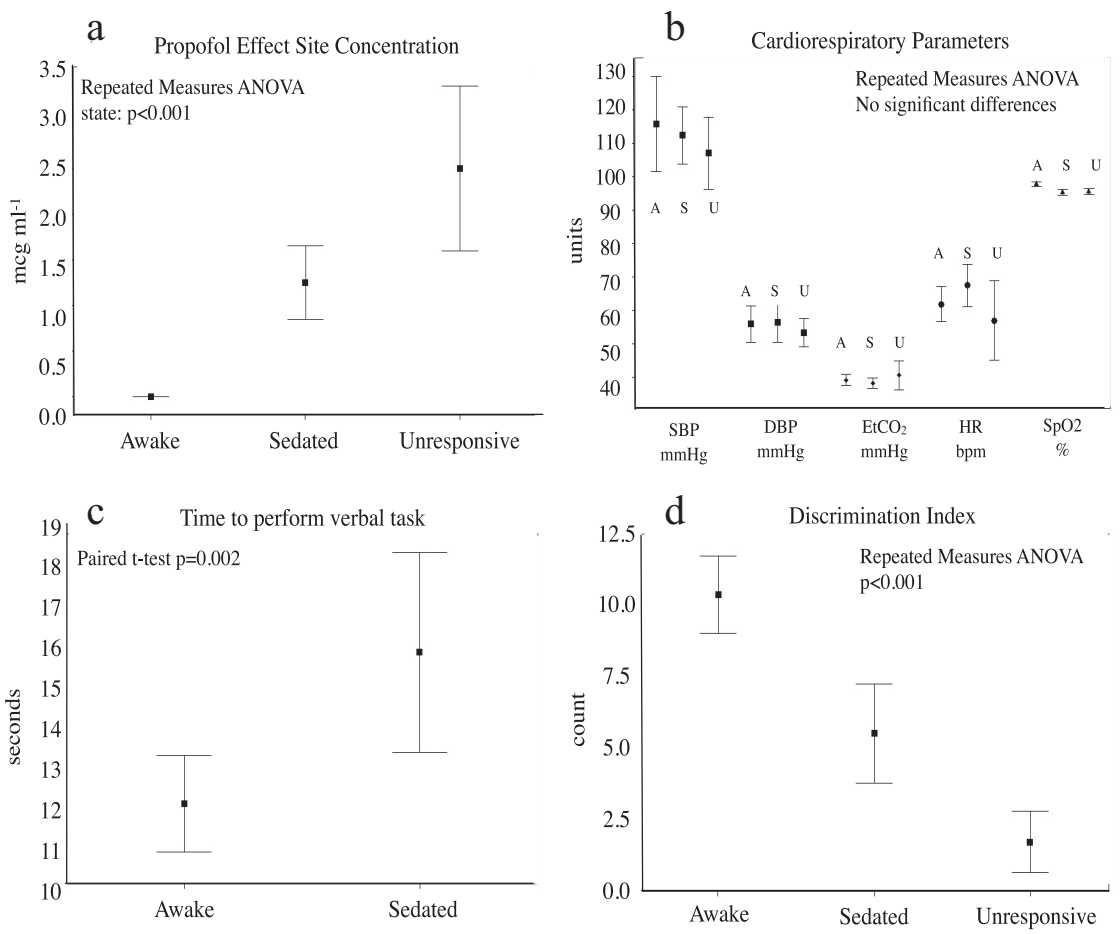

Figure 1. Physiology and psychophysical data. $\boldsymbol{a}$, Propofol effect site concentrations were significantly different across states (repeated-measures ANOVA, $p<0.001$ ). $\boldsymbol{b}$, There were not significant changes in physiological parameters across states. $\boldsymbol{c}$, Time to completion of the verbal task was significantly longer when sedated compared with awake $(p=0.002)$. $\boldsymbol{d}$, Discrimination index was significantly lower when sedated compared with awake $(p<0.001)$. A, Awake; S, sedated; $U$, unresponsive; SBP, systolic blood pressure; $\mathrm{DBP}$, diastolic blood pressure; $\mathrm{EtCO}_{2}$, end-tidal carbon dioxide; $\mathrm{HR}$, heart rate; $\mathrm{SpO}_{2}$, oxyhemoglobin saturation.

Discrimination index was assessed after each paradigm presentation, before the infusion rate was changed, except for the "unresponsive" state, where memory recall was tested at the end of the experiment when awake and in the recovery area.

Functional magnetic resonance imaging protocol. Volunteers were scanned in a 3 Tesla human Magnetic Resonance Imaging system, with a $1 \mathrm{~m}$ bore magnet (Oxford Magnet Technology Ltd), using a birdcage radio frequency coil and a reduced bore head gradient coil (Magnex SGRAD MKIII). The magnet was driven by a Varian Unity Inova console using Siemens' gradients.

A functional magnetic resonance whole brain gradient-echo echoplanar imaging sequence was used with the following parameters; Repetition time (TR) of $3000 \mathrm{~ms}$, echo time (TE) of $30 \mathrm{~ms}$, flip angle $=90^{\circ}$, field of view $=256$ by $256 \mathrm{~mm}$, matrix 64 by 64 . Twenty-one contiguous slices were taken through the whole brain with a slice thickness of $6 \mathrm{~mm}$. A 3D Turbo Flash T1-weighted (axial) high resolution anatomical scan was obtained at the end of the first experiment for each volunteer for coregistering the individual volunteer scans to a common standard template. Imaging parameters were $\mathrm{TR}=20 \mathrm{~ms}, \mathrm{TE}=4.6 \mathrm{~ms}$, flip angle $=$ 120, field of view 256 by $256 \mathrm{~mm}$ with a matrix of 256 by 256 and 64 contiguous slices of $3 \mathrm{~mm}$.

Data analysis. FMRI summary statistics, cardiorespiratory observations, discrimination index and propofol effect site concentration were analyzed using Minitab Statistical Software (Minitab Inc, PA). A p value of $<0.05$ was considered statistically significant. Paired $t$ tests and repeated measures ANOVA were used as appropriate.

FMRI data processing was performed using FEAT (FMRI Expert Analysis Tool) Version 5.98, part of FSL (FMRIB's Software Library, www.fmrib.ox.ac.uk/fsl).

Each fMRI acquisition consisted of 664 volumes. Four "dummy volumes" were deleted before further processing to ensure the signal had reached equilibrium.

The following preprocessing steps were applied to the raw data: motion correction parameters were estimated using MCFLIRT; signals from nonbrain areas were removed using BET (Brain Extraction Technique); spatial smoothing was applied to each fMRI volume using a Gaussian kernel of full-width half-maximum (FWHM) $5.0 \mathrm{~mm}$; lowfrequency artifacts were removed by applying a highpass temporal filter set at $0.01 \mathrm{~Hz}$.

Time series analysis of raw 4D fMRI data were performed for each session using FILM (FMRIB's Improved Linear Modeling). First level analysis of paradigm-related changes in blood oxygenation level-dependent (BOLD) signal identified areas within individual subjects' brains responding to specific stimuli. The general linear model for this analysis consisted of two explanatory variables: (1) timing of auditory stimuli and (2) timing of noxious stimuli, each convolved to the expected hemodynamic response function. Motion parameters were included as covariates of no interest. For each voxel, the higher the parameter estimate from the model fit, the better the data can be explained by the stimulus, implying activity in response to the stimulus. Second level analyses were performed using FEAT to estimate significant changes across the group in these parameter estimates (reflecting stimulus responses) due to propofol-induced changes in observed neurophysiological state.

A repeated-measures ANOVA was performed to identify areas where responses to each stimulus type varied significantly with neurophysiological state awake, impaired and unresponsive. To determine statistical significance of the observed activation while controlling for multiple comparisons, $Z$ statistic images were thresholded using clusters determined by $Z>2.3$ and a (corrected) cluster significance threshold of $p=0.05$ The directionality of the identified modulation was confirmed using paired $t$ tests.

For group analysis, a two step registration process was performed using FLIRT (FMRIB's Linear Registration Tool). Data were transformed first from functional space to individual subjects' structural space and then to a standard space (Montreal Neurological Institute MNI152 standard map).

Region-based connectivity analysis. To investigate changes in functional connectivity between brain regions, the individual subjects' mean BOLD signal time series from 13 regions of interest were extracted from each of the functional scans. Anatomical masks were hand drawn, without reference to functional activation maps, over the regions of interest. These masks, drawn in standard space, were then registered to each subject's functional space. Correlations between activation time series have a number of important limitations in the inferences that can be drawn. While they reflect a connectivity in terms of function, conclusions about the direction of inter-regional influences cannot be drawn. In addition, correlated changes in the ongoing data stream may indicate independent drive from a separate and perhaps common source, as noted when distinguishing "effective" from "functional" connectivity (Friston, 1994).

For each subject, mean time series consisting of the 660 BOLD signal intensities were than extracted for the following regions: hippocampus, Broca's area, amygdala, anterior cingulate cortex, posterior division of the superior temporal gyrus, primary somatosensory cortex, thalamus, putamen, pontine tegmentum, precuneus cortex, primary auditory cortex, substantia nigra and insular cortex. Qualitative estimation of functional connectivity changes was performed by calculating and plotting Pearson's correlation coefficients for pairs of regions of interest.

Whole-brain connectivity analysis. Two specific regions of interest were selected for further interrogation. The thalamus was chosen based on the extensive literature on its role in the neurophysiology of consciousness. The putamen was included due to the striking observation of changes in striatal responses to both types of stimulation (noxious and auditory) across states of consciousness. Statistical comparisons of the functional 
connectivity of both the thalamus and putamen were carried out, again using a generalized linear model within FEAT, with regressors at the first level derived this time from the mean signal time series from two specific regions of interest-thalamus and putamen. Multiplecomparisons constraints and significance thresholds were as for the stimulus-based analyses. Repeated-measures ANOVA across levels of sedation, identified areas of the brain where the functional connectivity to the thalamus and putamen was significantly altered by deepening sedation.

\section{Results}

\section{Physiology and psychophysical measures}

Physiology and psychophysical data reported pertain to the scanner session only. Propofol effect site concentrations differed significantly between states (repeated-measures ANOVA, $p<0.001$ ) (Fig. 1a). There was no significant difference in any of the cardiorespiratory parameters across conditions (Fig. 1b). The time required for completion of the verbal task was significantly longer (paired $t$ test, $p=0.002$ ) when "sedated" compared with "awake" (Fig. 1c). Discrimination index decreased significantly with increasing depth of sedation (repeated-measures ANOVA $p<0.001$ ) (Fig.

$1 d)$. There was no significant difference between pain ratings when the "awake" and "sedated" conditions were compared (paired $t$ test, $p=0.291)$.

\section{Stimulus-based analysis}

BOLD signal changes in response to binaurally presented auditory stimulation showed significant bilateral reductions in both cortical and subcortical regions with deepening sedation (Fig. 2, Table 1). The cortical regions identified were the anterior insula, anterior cingulate cortex and superior temporal gyrus, bilaterally. The significantly modulated subcortical structures were the putamen, pallidum and amygdala, again bilaterally.

BOLD signal changes in response to noxious thermal stimulation of the dorsum of the left hand showed significant bilateral reductions in the insula. However, the associated reductions in BOLD signal change in the putamen and globus pallidus were unilateral, and contralateral to the noxious stimulus.

Tables 1 and 2 give $Z$-scores and peak voxel coordinates of areas within the brain where the responses to auditory and noxious stimuli, respectively, decreased significantly with deepening sedation. Spatial maps of these results are shown in Figure 2.

\section{Connectivity analysis}

To illustrate the principle, Figure $3 a$ shows a 100 -volume sample of the averaged time series (i.e., averaged across voxels within the region in question and across all eight subjects) for three regions of interest at each level of sedation. This particular portion of the time series is shown because during this part of the stimulus presentation paradigm (thermal pain and tones), stimulus timings were identical for each subject, therefore information is not lost by averaging across subjects.

Expanding this to the 13 regions of interest interrogated, Figure $3 b$ illustrates the strength of the time series correlation be-
Table 1. Peak voxel coordinates: ANOVA of responses to auditory stimulation

\begin{tabular}{lrrrl}
\hline$Z$ & \multicolumn{1}{l}{$y$} & \multicolumn{1}{l}{$z$} & Region \\
\hline 4.29 & -34 & 20 & -4 & Right insula \\
4.29 & 40 & 21 & -4 & Left insula \\
4.66 & 62 & -32 & 5 & Right STG \\
4.34 & -59 & -34 & 2 & Left STG \\
3.44 & 22 & -1 & -13 & Right amygdala \\
3.1 & -25 & -1 & -13 & Left amygdala \\
3.59 & 4 & 18 & 34 & Right ACC \\
3.76 & -5 & 18 & 34 & Left ACC \\
4.26 & 22 & 5 & -2 & Right putamen \\
4.27 & -19 & 7 & -2 & Left putamen \\
4.1 & 15 & 5 & 0 & Right pallidum \\
3.7 & -16 & 3 & -3 & Left pallidum \\
\hline
\end{tabular}

STG, Superior temporal gyrus; ACC, anterior cingulate cortex.

Table 2. Peak voxel coordinates: ANOVA of responses to painful stimulation

\begin{tabular}{lrrrl}
\hline$Z$ & $x$ & $y$ & $z$ & Region \\
\hline 4.46 & 37 & 27 & 1 & Right insula \\
4.91 & -39 & 13 & 5 & Left insula \\
3.93 & 19 & 3 & 4 & Right putamen \\
3.34 & 17 & 2 & 0 & Right pallidum \\
\hline
\end{tabular}

tween regions and the modulation of this correlation which was found to occur with neurophysiological state change. We found that the correlation between the thalamus [red circle] and the regions of interest was minimally changed despite the clinical observations. The only exception was a reduction in connectivity between the thalamus and the putamen. While thalamic connectivity appeared to be thus preserved, we found a clear reduction in the strength of the correlation between the BOLD signal from the putamen [(blue circle)] and those same regions of interest. 
a
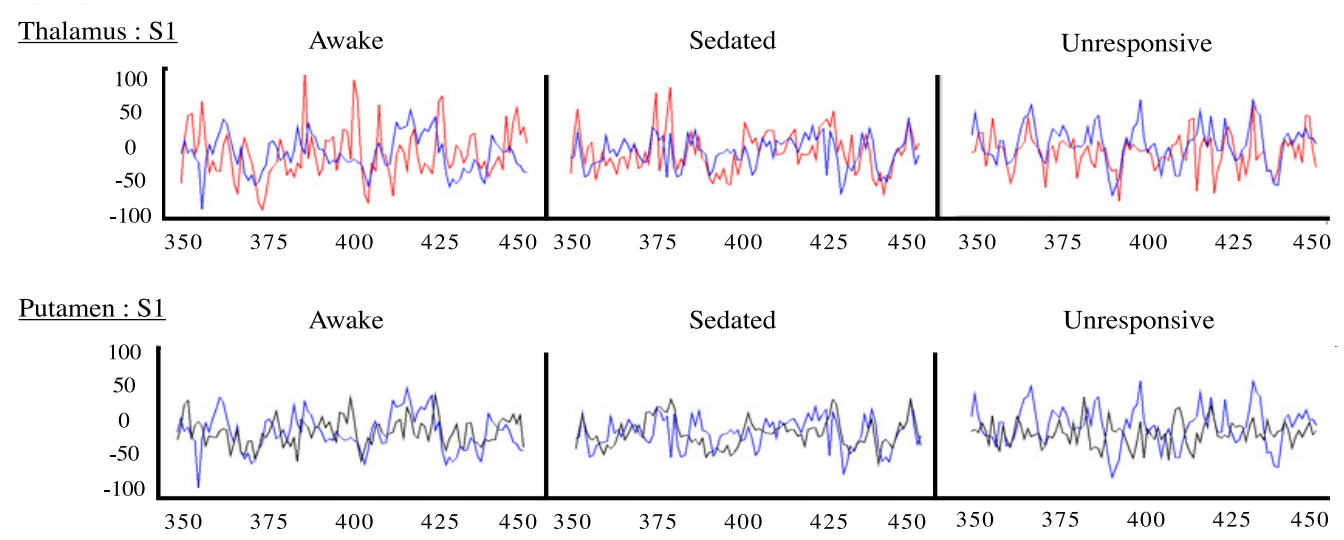

Putamen : Thalamus

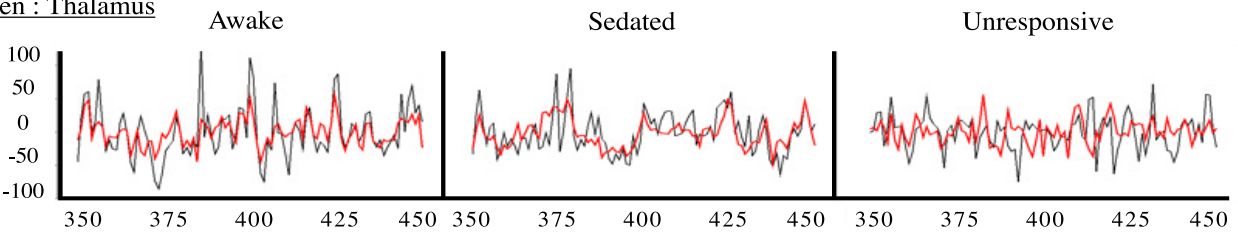

Correlation
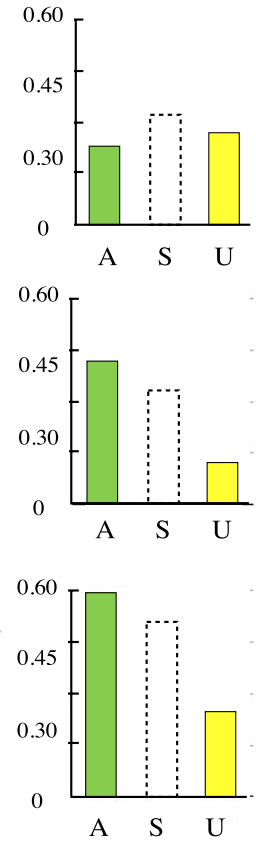

Thalamus Primary Somatosensory Cortex

Putamen

$\mathrm{b}$

Thalamus

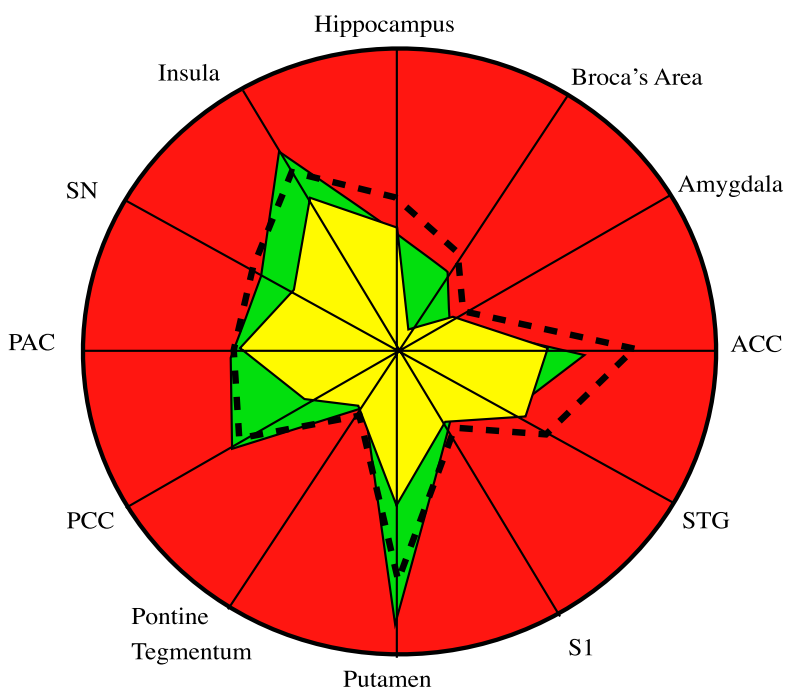

Awake

in ; Sedated

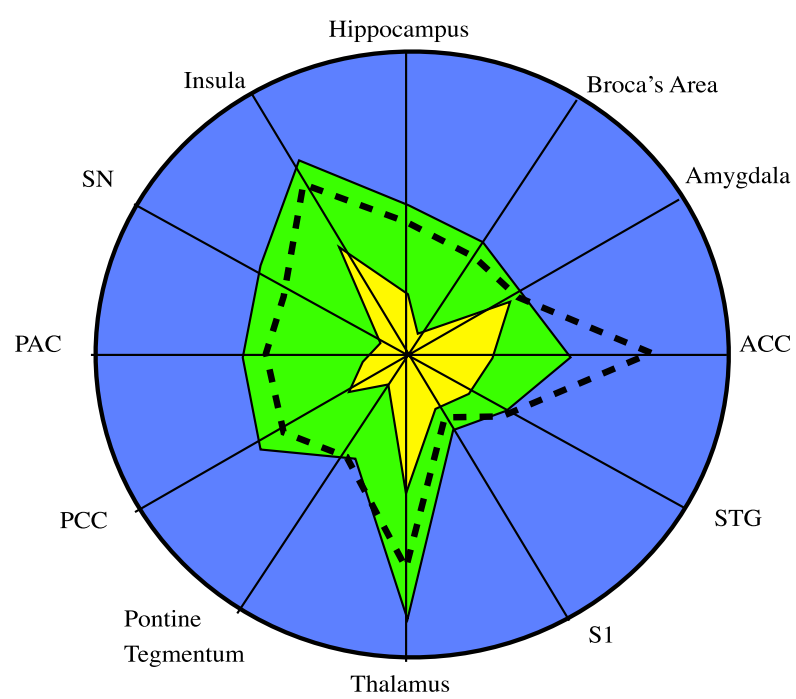

Unresponsive

Figure 3. Schematic illustrating functional connectivity changes of the putamen and thalamus. $\boldsymbol{a}$, Paired mean BOLD signals (averaged across subjects and region) from three regions of interest are shown in overlay. Plots show volumes $350-450$ (during which stimuli were presented at the same times to each subject) of 660 total volumes. Bar charts of the respective correlations were calculated across the entire 660 volume time series for individuals and then averaged. $\boldsymbol{b}$ illustrates the observed change in correlation, plotted on a unit circle, between the BOLD signal from the thalamus (left) and putamen (right) and each of 12 specific brain regions. S1, Primary somatosensory cortex; STG, superior temporal gyrus; ACC, anterior cingulate cortex; SN, substantia nigra; PAC, primary auditory cortex; $\mathrm{PCC}$, precuneus cortex.

This investigation provided a qualitative exploration of the data supporting the isolation of the putamen from other brain regions. Formal statistical evaluation of the suggested pattern of connectivity modulation was provided by the voxelwise fMRI analysis based on regressors derived from seed regions in the thalamus and putamen. The results of the repeated-measures ANOVA are shown in Figure 4. This gives a spatial map of those areas where connectivity was identified as being significantly altered across the defined states of consciousness. With respect to the thalamus, we found statistically significant decreases in the functional connectivity only with the putamen and a small region within the left posterior insula. However, statistically significant changes in functional connectivity with the putamen were widespread and confirmed the relative neurophysiological isolation of 
the putamen from other relevant structures, as suggested by our qualitative analysis of the signal correlation.

\section{Discussion}

Failure to perceive and remember external stimuli is one of the clinical endpoints of anesthesia, what the patient experiences. Loss of consciousness, the failure to respond to external stimuli, is what the witness observes. CNS changes underlying perceptual failure are poorly understood and the unreliability of the relationship between perceptual failure and observed response has driven the search for measures that better predict the subjective experience. Immobility and analgesia are not necessary for loss of consciousness but occur due to drug activity at widespread sites (Antognini et al., 2005). Similarly, analgesia is produced to varying degrees by different anesthetic agents and by nonanesthetic drugs, but is neither necessary nor sufficient for anesthesia (Sukhotinsky et al., 2007; Barter et al., 2008). Understanding the transition from awake to "unresponsive," and how various networks may be sequentially affected during this transition is key to dissecting this multidimensional phenomenon.

In our study, increasing levels of propofol produced the expected changes in behavior: a reduction in verbal task performance, increasing memory impairment and failure to respond. As significantly higher levels of propofol are required to produce surgical immobility than were used in our study (Milne et al., 2003), the behavioral state we defined as "unresponsive" is likely due to perceptual failure. We found this to be associated with changes in putamen connectivity rather than thalamic.

Hemodynamic changes can be a concern when analyzing the BOLD signal during drug manipulations, however, blood pressure and carbon dioxide level changes were nonsignificant (Fig. 1).

We confirmed expected reductions in stimulus-induced BOLD signal changes in cortical regions (Fig. 2). Observed changes in the response to auditory stimuli concur with published imaging studies investigating the effects of anesthetic agents on auditory-evoked brain activity, i.e., propofol-induced, dose-dependent reduction in higher auditory processing with preservation of activation in Heschl's gyrus (Dueck et al., 2005). With respect to nociceptive stimulation, it is interesting that areas normally showing attenuated BOLD responses with analgesia (thalamus, S1, anterior cingulate cortex, prefrontal cortex, posterior insula) were not attenuated. Propofol is however an anesthetic with weak if any analgesic action (TerRiet et al., 2000). Notably, no significant modulation of thalamic responses to either the auditory or thermal pain stimuli was observed.

What is striking however, is the significant reduction in activity within the putamen in response to both noxious and auditory stimuli as subjects transit toward the unresponsive state. Similar observations have been noted in other contexts by researchers investigating somatotopy within the striatum of anesthetized animals (West, 1998). They noted that both spontaneous and stimulus-induced activity within the striatum was exquisitely sensitive to different anesthetic agents. These changes occurred before changes in $\mathrm{S} 1$ responses.
The ANOVA produced two unexpected results with respect to regional responses to stimuli at increasing levels of propofol. Activation within the putamen decreased and activation in the thalamus did not. These structures may be differentially influenced by anesthetics and this was further interrogated with an analysis of functional connectivity. During the transition from awake to unresponsive, a clear reduction in functional connectivity between the putamen and a wide range of other cortical and subcortical regions occurred, while the connectivity of the thalamus with these regions was maintained. This preservation of thalamocortical connectivity has recently been observed in animals (Silva et al., 2010).

Studies of sleep and arousal have indicated that endogenous sleep promoting pathways may be final common targets for the diverse actions of anesthetic drugs (Campagna et al., 2003; Nelson et al., 2003; Franks, 2008; Lu et al., 2008). The common denominator in studies of both sleep and anesthesia is often the observation of reduced metabolic activity and/or blood flow within the thalamus. These observations have contributed to the concept that the thalamus is pivotal in producing sleep or an unconscious state. As a model of anesthesia, however, physiological sleep is incomplete due to preservation of protective arousal. Failure of arousal upon intense sensory input during anesthesia limits the parallels which can be drawn. Disordered or failed perception is however seen in a number of pathological conditions, consideration of which may form the basis of useful alternative analogues.

Gross CNS pathologies (e.g., hemispherectomy) can coexist with the preservation of consciousness (Fountas et al., 2006), while small lesions affecting specific regions can impair consciousness, perception and/or generation of organized motor responses. Minimally conscious states may occur with thalamic strokes affecting the reticular (RT) or intralaminar (ILN) thalamic nuclei or lesions of the reticular formation (Schiff and Plum, 2000). Described formerly as "nonspecific" thalamic nuclei, the anterior and posterior intralaminar nuclei of the thalamus together with the paralaminar portions of the thalamic 
association nuclei are recognized as central to consciousness (Schiff, 2008). These nuclei project to both the cortex and striatum (Herrero et al., 2002; Behrens et al., 2003).

A key anatomical specialization of the intralaminar nuclei is that they comprise the largest thalamic efference to the striatum, an important pathway in coordination of thalamocortical signal processing (Schiff and Plum, 2000). Our observations suggest that the putamen is particularly sensitive to propofol and that before the failure of thalamocortical transmission, at the point at which subjects fail to perceive and lose verbal contact, the thalamo-regulatory influence of cortico-striato-thalamo-cortical circuits may be disrupted. While we failed to detect a change in thalamocortical connectivity with respect to an averaged BOLD signal from the thalamus, individual nuclei may be independently modulated. This is significant in the context of the expected association of thalamocortical disconnection with unresponsive states. Further imaging studies at higher field strength may unmask such changes in primary and secondary thalamic nuclei although the gross anatomy of the critical ILN and RN makes noninvasive interrogation challenging.

The reduced thalamic activity reported under anesthesia may reflect a direct action on thalamic nuclei inhibiting thalamocortical transmission. Alternatively, reduced excitatory input to the thalamus from other sites may lead to the observation of reduced local blood flow and metabolism (Tononi and Edelman, 1998; White and Alkire, 2003). Tononi and Edelman propose that activation and deactivation of distributed neural populations in the thalamocortical system is probably not itself a sufficient basis for conscious experience unless activity of the relevant neuronal groups subserving that experience are rapidly and effectively integrated. They suggest that such rapid integration is achieved via "reentry"; ongoing, recursive and highly parallel signaling within and among brain areas considered essential for conscious experience.

It is perhaps unsurprising to find that the putamen is specifically vulnerable to lower doses of anesthetics, considering the frequency with which transient dystonic movements are observed during the induction of anesthesia (Walder et al., 2002). The striatum is not exclusively motor however, but plays an integral role in coordination of both afferent and efferent neuronal signals. Basal ganglia structures are not commonly reported in the acute pain literature (Jones et al., 1991; Casey et al., 1994) but involvement in the processing of nociceptive information (Tracey et al., 2000), including attention to noxious information, motor preparation and response selection are increasingly recognized, and dedicated nociceptive pathways to the striatum have been identified (Braz et al., 2005). Iardola and colleagues have observed bilateral activation of the putamen with capsaicininduced pain and the basal ganglia have also been found to play a role in chronic pain conditions such as atypical facial pain (Hagelberg et al., 2003a) and burning mouth syndrome (Hagelberg et al., 2003b). The reader is referred to an excellent review for further consideration of the role of the basal ganglia in pain perception (Chudler and Dong, 1995).

Di Martino reported a resting state fMRI study in humans demonstrating functional organization consistent with the existence of parallel loops through the basal ganglia subserving the multiple integrative functions as required by the construct described by Tononi and colleagues for conscious perception (Tononi and Edelman, 1998; Di Martino et al., 2008). Our experiment cannot distinguish between direct propofol modulation of the striatum itself and striatal deafferentation secondary to drug activity at another site such as the cortex, brainstem or indeed the thalamus (Abulafia et al., 2009). This is a parallel problem to that raised by Bonhomme and colleagues, who found cortical activation to be reduced at lower doses than those required to reduce activation within the thalamus (Bonhomme et al., 2001).

Cortical activity constitutes the contents of consciousness but the state of consciousness, perception of those contents, requires their dynamic integration into experience (cognitive binding) and the generation of appropriate responses (motor, cognitive, affective, memory formation) (Baars, 1995). Integration is dependent upon positive feedback loops facilitating the transmission of relevant signals through the thalamus. Cortical afferents to the thalamus travel via the striatopallidal fibers, and reversible thalamic suppression due to deafferentation has been described (Schiff et al., 2007). Basal ganglia circuits are involved in regulating signal transmission from thalamus to cortex. We have shown that levels of propofol producing sedation but not surgical anesthesia, impair the function of these circuits before thalamocortical connectivity is interrupted.

\section{References}

Abulafia R, Zalkind V, Devor M (2009) Cerebral activity during the anesthesia-like state induced by mesopontine microinjection of pentobarbital. J Neurosci 29: 7053-64.

Alkire MT (2008) Loss of effective connectivity during general anesthesia. Int Anesthesiol Clin 46:55-73.

Alkire MT, Pomfrett CJ, Haier RJ, Gianzero MV, Chan CM, Jacobsen BP, Fallon JH (1999) Functional brain imaging during anesthesia in humans: effects of halothane on global and regional cerebral glucose metabolism. Anesthesiology 90:701-709.

Alkire MT, Haier RJ, Fallon JH (2000) Toward a unified theory of narcosis: brain imaging evidence for a thalamocortical switch as the neurophysiologic basis of anesthetic-induced unconsciousness. Conscious Cogn 9:370-386.

Alkire MT, McReynolds JR, Hahn EL, Trivedi AN (2007) Thalamic microinjection of nicotine reverses sevoflurane-induced loss of righting reflex in the rat. Anesthesiology 107:264-272.

Angel A (1991) The G. L. Brown lecture. Adventures in anesthesia. Exp Physiol 76:1-38.

Antognini JF, Schwartz K (1993) Exaggerated anesthetic requirements in the preferentially anesthetized brain. Anesthesiology 79:1244-1249.

Antognini JF, Barter L, Carstens E (2005) Overview movement as an index of anesthetic depth in humans and experimental animals. Comp Med 55:413-418.

Baars BJ (1995) Tutorial commentary: surprisingly small subcortical structures are needed for the state of waking consciousness, while cortical projection areas seem to provide perceptual contents of consciousness. Conscious Cogn 4:159-162.

Barter LS, Mark LO, Jinks SL, Carstens EE, Antognini JF (2008) Immobilizing doses of halothane, isoflurane or propofol, do not preferentially depress noxious heat-evoked responses of rat lumbar dorsal horn neurons with ascending projections. Anesth Analg 106:985-990.

Behrens TE, Johansen-Berg H, Woolrich MW, Smith SM, Wheeler-Kingshott CA, Boulby PA, Barker GJ, Sillery EL, Sheehan K, Ciccarelli O, Thompson AJ, Brady JM, Matthews PM (2003) Noninvasive mapping of connections between human thalamus and cortex using diffusion imaging. Nat Neurosci 6:750-757.

Biswal B, Yetkin FZ, Haughton VM, Hyde JS (1995) Functional connectivity in the motor cortex of resting human brain using echo-planar MRI. Magn Reson Med 34:537-541.

Bonhomme V, Fiset P, Meuret P, Backman S, Plourde G, Paus T, Bushnell MC, Evans AC (2001) Propofol anesthesia and cerebral blood flow changes elicited by vibrotactile stimulation: a positron emission tomography study. J Neurophysiol 85:1299-1308.

Braz JM, Nassar MA, Wood JN, Basbaum AI (2005) Parallel "pain” pathways arise from subpopulations of primary afferent nociceptor. Neuron 47:787-793.

Campagna JA, Miller KW, Forman SA (2003) Mechanisms of actions of inhaled anesthetics. N Engl J Med 348:2110-2124.

Casey KL, Minoshima S, Berger KL, Koeppe RA, Morrow TJ, Frey KA (1994) Positron emission tomographic analysis of cerebral structures acti- 
vated specifically by repetitive noxious heat stimuli. J Neurophysiol 71:802-807.

Chudler EH, Dong WK (1995) The role of the basal ganglia in nociception and pain. Pain 60:3-38.

Detsch O, Vahle-Hinz C, Kochs E, Siemers M, Bromm B (1999) Isoflurane induces dose-dependent changes of thalamic somatosensory information transfer. Brain Res 829:77-89.

Devor M, Zalkind V (2001) Reversible analgesia, atonia, and loss of consciousness on bilateral intracerebral microinjection of pentobarbital. Pain 94:101-112.

Di Martino A, Scheres A, Margulies DS, Kelly AM, Uddin LQ, Shehzad Z, Biswal B, Walters JR, Castellanos FX, Milham MP (2008) Functional connectivity of human striatum: a resting state FMRI study. Cereb Cortex 18:2735-2747.

Dueck MH, Petzke F, Gerbershagen HJ, Paul M, Hesselmann V, Girnus R, Krug B, Sorger B, Goebel R, Lehrke R, Sturm V, Boerner U (2005) Propofol attenuates responses of the auditory cortex to acoustic stimulation in a dose-dependent manner: a FMRI study. Acta Anaesthesiol Scand 49:784-791.

Fiset P, Paus T, Daloze T, Plourde G, Meuret P, Bonhomme V, Hajj-Ali N, Backman SB, Evans AC (1999) Brain mechanisms of propofol-induced loss of consciousness in humans: a positron emission tomographic study. J Neurosci 19:5506-5513.

Fountas KN, Smith JR, Robinson JS, Tamburrini G, Pietrini D, Di Rocco C (2006) Anatomical hemispherectomy. Childs Nerv Syst 22:982-991.

Franks NP (2008) General anesthesia: from molecular targets to neuronal pathways of sleep and arousal. Nat Rev Neurosci 9:370-386.

Friston K (1994) Functional and effective connectivity in neuroimaging: a synthesis. Hum Brain Mapp 2:56-78.

Gepts E, Camu F, Cockshott ID, Douglas EJ (1987) Disposition of propofol administered as constant rate intravenous infusions in humans. Anesth Analg 66:1256-1263.

Gusnard DA, Raichle ME (2001) Searching for a baseline: functional imaging and the resting human brain. Nat Rev Neurosci 2:685-694.

Hagelberg N, Forssell H, Aalto S, Rinne JO, Scheinin H, Taiminen T, Någren K, Eskola O, Jääskeläinen SK (2003a) Altered dopamine D2 receptor binding in atypical facial pain. Pain 106:43-48.

Hagelberg N, Forssell H, Rinne JO, Scheinin H, Taiminen T, Aalto S, Luutonen S, Någren K, Jääskeläinen S (2003b) Striatal dopamine D1 and D2 receptors in burning mouth syndrome. Pain 101:149-154.

Herrero MT, Barcia C, Navarro JM (2002) Functional anatomy of thalamus and basal ganglia. Childs Nerv Sys 18:386-404.

Jones AK, Brown WD, Friston KJ, Qi LY, Frackowiak RS (1991) Cortical and subcortical localization of response to pain in man using positron emission tomography. Proc Biol Sci 244:39-44.

Kaisti KK, Metsähonkala L, Teräs $M$, Oikonen V, Aalto $S$, Jääskeläinen S, Hinkka S, Scheinin H (2002) Effects of surgical levels of propofol and sevoflurane anesthesia on cerebral blood flow in healthy subjects studied with positron emission tomography. Anesthesiology 96:1358-1370.

Laitio RM, Kaisti KK, Låangsjö JW, Aalto S, Salmi E, Maksimow A, Aantaa R, Oikonen V, Sipilä H, Parkkola R, Scheinin H (2007) Effects of xenon anesthesia on cerebral blood flow in humans: a positron emission tomography study. Anesthesiology 106:1128-1133.

Laitio RM, Långsjö JW, Aalto S, Kaisti KK, Salmi E, Maksimow A, Aantaa R, Oikonen V, Viljanen T, Parkkola R, Scheinin H (2009) The effects of xenon anesthesia on the relationship between cerebral glucose metabolism and blood flow in healthy subjects: a positron emission tomography study. Anesth Analg 108:593-600.

Lu J, Nelson LE, Franks N, Maze M, Chamberlin NL, Saper CB (2008) Role of endogenous sleep-wake and analgesic systems in anesthesia. J Comp Neurol 508:648-662.

Marsh B, White M, Morton N, Kenny GN (1991) Pharmacokinetic model driven infusion of propofol in children. Br J Anaesth 67:41-48.

Mashour GA (2006) Integrating the science of consciousness and anesthesia. Anesth Analg 103:975-982.

Massimini M, Ferrarelli F, Huber R, Esser SK, Singh H, Tononi G (2005)
Breakdown of cortical effective connectivity during sleep. Science 309:2228-2232.

Meyer HM (1899) Zur Theorie der Alkoholnarkose. Arch Exp Pathol Pharmakol 42:109-118.

Milne SE, Troy A, Irwin MG, Kenny GN (2003) Relationship between bispectral index, auditory evoked potential index and effect-site EC50 for propofol at two clinical end-points. Br J Anaesth 90:127-131.

Nelson LE, Guo TZ, Lu J, Saper CB, Franks NP, Maze M (2002) The sedative component of anesthesia is mediated by GABA(A) receptors in an endogenous sleep pathway. Nat Neurosci 5:979-984.

Nelson LE, Lu J, Guo T, Saper CB, Franks NP, Maze M (2003) The alpha2adrenoceptor agonist dexmedetomidine converges on an endogenous sleep-promoting pathway to exert its sedative effects. Anesthesiology 98:428-436.

Prys-Roberts C (1987) Anesthesia: a practical or impractical construct? Br J Anesth 59:1341-1345.

Rasmusson DX, Bylsma FW, Brandt J (1995) Stability of performance on the Hopkins Verbal Learning Test. Arch Clin Neuropsychol 10:21-26.

Schiff ND (2008) Central thalamic contributions to arousal regulation and neurological disorders of consciousness. Ann N Y Acad Sci 1129:105-118.

Schiff ND, Plum F (2000) The role of arousal and "gating" systems in the neurology of impaired consciousness. J Clin Neurophysiol 17:438-452.

Schiff ND, Giacino JT, Kalmar K, Victor JD, Baker K, Gerber M, Fritz B, Eisenberg B, Biondi T, O'Connor J, Kobylarz EJ, Farris S, Machado A, McCagg C, Plum F, Fins JJ, Rezai AR (2007) Behavioural improvements with thalamic stimulation after severe traumatic brain injury. Nature 448:600-603.

Sear JW (2009) What makes a molecule an anaesthetic? Studies on the mechanisms of anesthesia using a physicochemical approach. Br J Anaesth 103:50-60.

Shapiro AM, Benedict RH, Schretlen D, Brandt J (1999) Construct and concurrent validity of the Hopkins Verbal Learning Test-revised. Clin Neuropsychol 13:348-358.

Silva A, Cardoso-Cruz H, Silva F, Galhardo V, Antunes L (2010) Comparison of anesthetic depth indexes based on thalamocortical local field potentials in rats. Anesthesiology 112:355-363.

Sukhotinsky I, Zalkind V, Lu J, Hopkins DA, Saper CB, Devor M (2007) Neural pathways associated with loss of consciousness caused by intracerebral microinjection of GABA A-active anesthetics. Eur J Neurosci 25:1417-1436.

TerRiet MF, Jacobs JS, Lewis MC, DeSouza GJ (2000) Propofol and analgesia. Anesth Analg 90:1455.

Tononi G, Edelman GM (1998) Consciousness and complexity. Science 282:1846-1851.

Tracey I, Becerra L, Chang I, Breiter H, Jenkins L, Borsook D, González RG (2000) Noxious hot and cold stimulation produce common patterns of brain activation in humans: a functional magnetic resonance imaging study. Neurosci Lett 288:159-162.

Van der Werf YD, Witter MP, Groenewegen HJ (2002) The intralaminar and midline nuclei of the thalamus. Anatomical and functional evidence for participation in processes of arousal and awareness. Brain Res Brain Res Rev 39:107-140.

Veselis RA, Feshchenko VA, Reinsel RA, Dnistrian AM, Beattie B, Akhurst TJ (2004) Thiopental and propofol affect different regions of the brain at similar pharmacologic effects. Anesth Analg 99:399-408.

Vincent JL, Patel GH, Fox MD, Snyder AZ, Baker JT, Van Essen DC, Zempel JM, Snyder LH, Corbetta M, Raichle ME (2007) Intrinsic functional architecture in the anaesthetized monkey brain. Nature 447:83-86.

Walder B, Tramèr MR, Seeck M (2002) Seizure-like phenomena and propofol: a systematic review. Neurology 58:1327-1332.

West MO (1998) Anesthetics eliminate somatosensory-evoked discharges of neurons in the somatotopically organized sensorimotor striatum of the rat. J Neurosci 18:9055-9068.

White NS, Alkire MT (2003) Impaired thalamocortical connectivity in humans during general-anesthetic-induced unconsciousness. Neuroimage 19:402-411. 